\title{
Symmetry of evaluation in the reinforcement-attraction paradigm
}

\author{
FRANCIS S. GILBERT \\ Purdue University, West Lafayette, Indiana 47907
}

\begin{abstract}
Page and Moss (1975) have noted that, while attitude similarity has been found to lead to high attraction, attitude dissimilarity has generally been found to lead to only slight disliking. Page and Moss (1975) hypothesized that this asymmetry was the result of a social desirability artifact, but were able to provide only partial support for the hypothesis. It was hypothesized herein that asymmetry of evaluation was a result of limitations built into the usual attraction measurement procedures. When a multidimensional, unipolar scale was substituted for the typical unidimensional, bipolar scale, liking and disliking were indeed found to be symmetrical.
\end{abstract}

In a recent article, Page and Moss (1975) pointed out that studies conducted within the reinforcementattraction paradigm (Byrne, 1971) contain an incongruity with regard to the prediction of attraction from proportion of positive reinforcements. That is, in the linear equation for predicting attraction from proportion of similar attitudes (Byrne \& Nelson, $1965)^{1}$, there is an unexplained asymmetry; i.e., fairly high attraction is predicted under conditions of $100 \%$ reinforcement $(\hat{Y}=12.06)$, but only slight disliking is predicted by the complete absence of reinforcement $(\hat{Y}=6.62)$. Page and Moss (1975) hypothesized that this asymmetry was due to differences in the properties of reinforcement and punishment or, alternatively, to subjects' hesitancies to evaluate a stranger unfavorably.

Page and Moss (1975) tested the second hypothesis by comparing measurements of liking obtained with the typical Interpersonal Judgement Scale (IJS) rating procedure (Byrne, 1971) with those obtained with a bogus pipeline procedure. It was predicted that the bogus pipeline procedure, which was designed to decrease social desirability artifacts by convincing subjects that the experimenter had a physiological measure of their true feelings (Jones \& Sigall, 1971), would eliminate reluctance to express negative feelings. With social desirability response biases attenuated, symmetry of like and dislike were predicted in the bogus pipeline condition. Their results, however, provided only partial support for their hypothesis. In only one of the seven analyses conducted was evidence found for the predicted interaction between similarity and measurement condition.

Although Page and Moss (1975) provided some support for the presence of a social desirability

This paper is sponsored by D. Byrne, who takes full editorial responsibility. Requests for reprints should be sent to Donn Byrne, Department of Psychological Sciences, Purdue University, West Lafayette, Indiana 47907.

artifact affecting measurement in the similarityattraction methodology, a recent study (Cherry, Byrne, \& Mitchell, 1976) has demonstrated that: (1) the bogus pipeline procedure is not superior to rating scale techniques for measuring interpersonal attraction, and (2) the similarity-attraction effect is not altered by demand characteristics when the typical rating procedure is used. Based upon these results (Cherry et al., 1976), it is unlikely that asymmetry of evaluative responding is the result of a social desirability bias.

An alternative hypothesis for the asymmetry phenomenon is that asymmetry exists because the IJS does not measure dislike. The assumption which underlies the structure of the IJS is that liking and disliking responses constitute opposite ends of a single continuum. An alternative assumption, and the one favored by the author, is that evaluation consists of two components, i.e., independent like and dislike responses. If so, then evaluation should be measured by multidimensional, unipolar response measures instead of a unidimensional, bipolar measure. The importance of this distinction is that the IJS, which utilizes a bipolar response format, might be requiring subjects to weigh the amount they like a stranger against the amount they dislike him or her. Measurement would then be of net evaluation rather than gross liking and gross disliking. The result would be that the IJS would not measure dislike at all, and symmetrical like and dislike responses would be impossible to obtain.

Indirect support for this prediction does exist. Gouaux, Lamberth, and Friedrich (1972) had subjects evaluate themselves, their best friend, and the person they liked least on the First-Impression Rating Scale, Form I (Izard \& Nunnally, 1965). Subjects then took part in a similarity manipulation study and completed the IJS for a bogus stranger. Correlations between the First-Impression Rating Scales (self and best friend) and the IJS were found to be significant regardless of whether the First- 


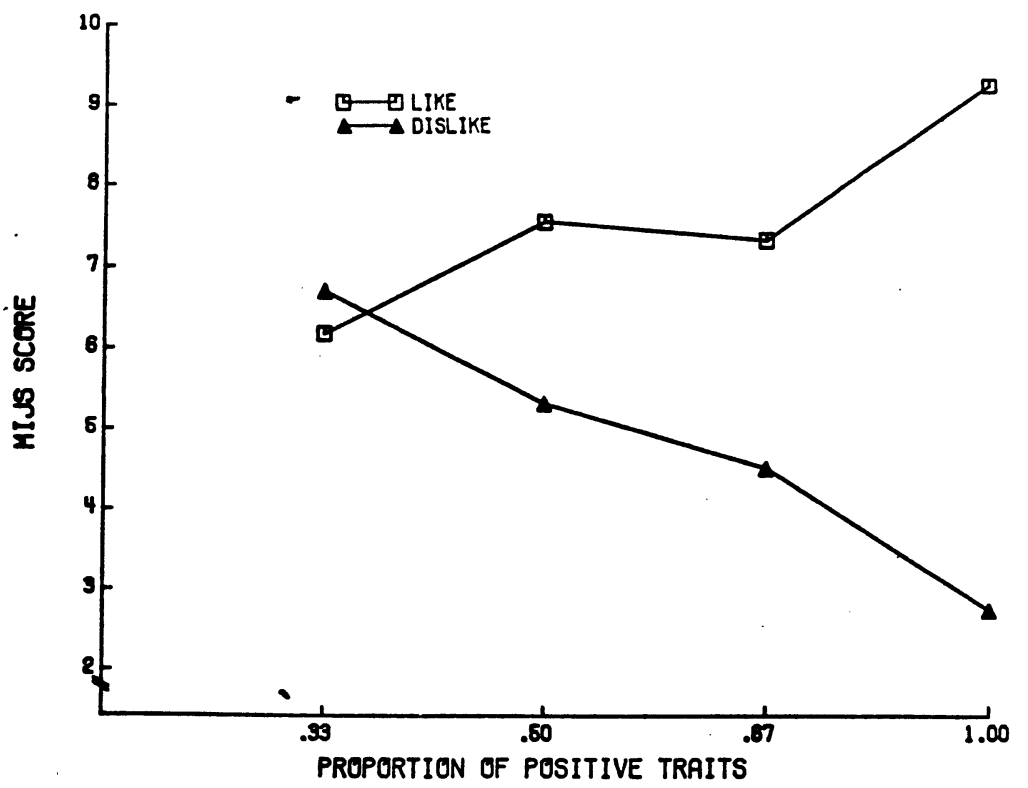

Figure 1. Evaluative responding as a function of proportion of positive feedback.

Impression Rating Scales had been obtained 2 months prior to the experiment ( $\mathrm{rs}=.27$ and .20 , respectively, ps <.05) or at the time of the experiment (rs = .45 and .22 , respectively, ps $<.01$ ). The FirstImpression Rating Scale evaluations of the least liked person did not correlate with the IJS whether collected prior to the experiment $(r=.03)$ or at the time of the experiment $(r=.09)$, however. The latter correlations indicate the absence of a relationship between a disliking response and the IJS, as suggested.

In the present experiment it was predicted that, if asymmetry is the result of measurement limitations built into the IJS, a scale which measured like and dislike independently would exhibit symmetrical evaluations under $100 \%$ and $0 \%$ positive reinforcement conditions.

\section{METHOD}

\section{Overview and Subjects}

The relationship between evaluative symmetry and reinforcement was investigated in a $\mathbf{3}$ by $\mathbf{4}$ factorial design using a bidimensional, unipolar dependent variable. The design was a systematic replication of Byrne and Nelson (1965), the only difference being the use of positive and negative evaluations as stimuli in place of similar and dissimilar attitudes. Three numbers of reinforcers $(4,8$, and 16) were crossed with four proportions of positive and negative reinforcement $(.33, .50, .67,1.00)$. Evaluative feedback consisted of adjectives from Anderson's (1968) list of personality traits. The scale values provided by Anderson (1968) were used to equate the overall favorability of the eight lists of traits required by the design. Subjects were 144 introductory psychology students who volunteered to participate in the experiment to fulfill a class requirement.

\section{Dependent Measure}

To measure liking and disliking independently, a Modified Interpersonal Judgement Scale (MUS) was developed. The "personal feelings" and "working together" items of the IJS were converted to a unipolar format to form a liking scale with a range of absence of liking (e.g., "I do not feel that I would like this person") to much liking (e.g., "I believe that I would like working with this person in an experiment immensely"). Two new items (voting in an election and developing a friendship) were added to form a disliking scale ranging from the absence of disliking (e.g., not difficult to establish a friendship with this person) to much disliking (e.g., immensely opposed to this person's election). Five intermediate steps were included in each item, ranging from "slightly" to "a very great deal." The four filler items from the IJS were retained in the MIJS in their original format.

\section{Procedure}

Subjects participated in the experiment in groups of 12 . During the experiment each subject was assigned to an individual booth equipped with a headset, microphone, and television monitor. Subjects were instructed over an intercom system that they would be presented with a series of social situations. During the first part of the experiment, half of the subjects were to respond to the situation (speaker's role) and half were to listen to those responses (listener's role). Each speaker was purported to be speaking to only one listener, and each listener to be listening to only one speaker. Based upon the speaker's responses to the situations, each listener was then to evaluate the person he or she had listened to on a personality rating scale. After he informed the subjects as a group that he randomly would assign them to the speaker's role or the listener's role, the experimenter proceeded to assign individually every subject to the speaker's role. Unbeknownst to the subjects, the intercom system connecting the rooms was then turned off, and all responses made by the subjects were made only to themselves.

The stimulus situations were eight videotaped vignettes read by either a male or female assistant (depending upon the subjects' sex), and played over the in-booth monitors. All of the vignettes employed a heterosexual interaction theme. For example, "Let's say you're at a bar sitting alone at a table. An attractive guy [girl] comes over and you each introduce yourselves. You say, 'Would you like to sit down?' He [she] looks over and smiles at you. You say ... ."Following the "You say" cue, the tape went blank and subjects had $20 \mathrm{sec}$ in which to role play their response to the situation. After the final stimulus situation, all subjects heard the experimenter tell the "listeners" that he would now pass out the personality rating scale. Following the short break necessitated by the deception, a bogus personality rating scale was given to each subject. After examining the feedback, each subject completed a MIJS.

A group debriefing session followed collection of the MUS. 


\section{RESULTS}

Each of the evaluation items was scored from one to seven, and the respective sums of the liking and disliking items provided the basic data for analysis. Each dependent variable was analyzed separately. On both scales, the only significant effect was the proportion of positive feedback variable $(\mathrm{Fs}=11.22$ and 15.16 for liking and disliking, respectively, ps $<.01)$. Thus, the familiar relationship between proportion of positive reinforcement and attraction, developed with a bipolar scale, is equally apparent with two unipolar dependent variables. The means for both scales at each level of proportion of positive feedback are shown in Figure 1.

To test the prediction of symmetry, the 144 liking and disliking scores were divided into two subsamples of 72 subjects each. The liking and disliking scores for each of these subsamples were then separately regressed onto the proportion of positive feedback variable. This yielded two independent sets of two regression equations, viz., one for predicting liking and one for predicting disliking from each subsample. Symmetry was tested by cross-sample comparisons of the absolute values of the regression coefficients. All four comparisons yielded nonsignificant differences between the coefficients of the two samples (ps $>.25) .{ }^{2}$ Thus, when evaluation was measured on a bidimensional, unipolar scale, the amount of change in both responses, per unit of change in proportion of reinforcement, was equal. In other words, the two evaluative responses were symmetrical, as predicted.

\section{DISCUSSION}

The results of this study support the hypothesis that the asymmetry of the Byrne and Nelson (1965) regression equation is due to the assumptions underlying the measurement of attraction with the IJS. When a multidimensional, unipolar scale of evaluation such as the MUS is used, liking and disliking are indeed symmetrical.

\section{NOTES}

1. $\hat{Y}=5.44 X+6.62$.

2. Because there were no differences between the two subsamples, they were collapsed and regression equations for like and dislike were computed on the entire sample. These equations were: $\hat{\mathrm{L}}=4.61+4.71$ and $\hat{\mathrm{D}}=-5.19+8.03$.

\section{REFERENCES}

ANDERson, N. H. Likableness ratings of 555 personality-trait words. Journal of Personality and Social Psychology, 1968, 9, 272-279.

BYRNE, D. The attraction paradigm. New York: Academic Press, 1971.

Byrne, D., \& Nelson, D. Attraction as a linear function of proportion of positive reinforcements. Journal of Personality and Social Psychology, 1965, 1, 659-663.

Cherry, F., Byrne, D., \& Mitchell, H. E. Clogs in the bogus pipeline: Demand characteristics and social desirability. Journal of Research in Personality, 1976, 10, $69-75$

Gouaux, C., Lamberth, J., \& Friedrich, G. Affect and interpersonal attraction: A comparison of trait and state measures. Journal of Personality and Social Psychology, 1972, 25, 53-58.

Izard, C. E., \& Nunnally, J. C. Evaluative responses to affectively positive and negative facial photographs: Facial structure and construct validity. Educational and Psychological Measurement, 1965, 25, 1061-1071.

Jones, E. E., \& Sigall, H. The bogus pipeline: A new paradigm for measuring affect and attitude. Psychological Bulletin, 1971, 76, 349-364.

PAGe, R. A., \& Moss, M. K. Attitude similarity and attraction: The effects of the bogus pipeline. Bulletin of the Psychonomic Society, 1975, 5, 63-65.

(Received for publication June 28, 1976.) 